\title{
Quaderni
}

QUADERNI Communication, technologies, pouvoir

76 | Automne 2011

Les promesses de la biodiversité

\section{Gouverner par les scénarios? Comment les institutions environnementales anticipent l'avenir de la biodiversité}

Avant-propos

Céline Granjou et Isabelle Mauz

\section{OpenEdition}

Journals

\section{Édition électronique}

URL : http://journals.openedition.org/quaderni/72

DOI : 10.4000 /quaderni.72

ISSN : 2105-2956

Éditeur

Les éditions de la Maison des sciences de l'Homme

Édition imprimée

Date de publication : 1 septembre 2011

Pagination : 5-11

\section{Référence électronique}

Céline Granjou et Isabelle Mauz, « Gouverner par les scénarios ? Comment les institutions

environnementales anticipent l'avenir de la biodiversité », Quaderni [En ligne], 76 | Automne 2011, mis en ligne le 01 septembre 2011, consulté le 10 décembre 2020. URL : http://journals.openedition.org/ quaderni/72 ; DOI : https://doi.org/10.4000/quaderni.72 


\section{$D$ ossier}

avant-propos : gouverner par les scénarios?

Comment les institutions

environnementales anticipent l'avenir de la biodiversité

\section{Céline Granjou}

Chargée de recherches en sociologie au Cemagref de Grenoble

Isabelle
Mauz

Chercheure en sociologie au Cemagref de Grenoble
2010 a été déclarée " Année internationale de la biodiversité ». La mise à l'agenda des préoccupations relatives à l'érosion de la biodiversité n'est pourtant pas récente. Dès les années 1980, cette notion a été mise en avant par des scientifiques militants désireux de susciter la prise de conscience des autorités politiques (Takacs, 1996). La Convention sur la Diversité Biologique a été créée en 1992. Dans le courant des années 2000, nombre de pays européens, dont la France en 2004, se sont dotés d'une stratégie nationale pour la biodiversité. L'objectif de réduire l'érosion de la biodiversité à l'horizon 2010 a été fixé mais, faute d'avoir été atteint, il a été repoussé à 2020. Aujourd'hui, de nouvelles institutions ont été mises en place ou sont en voie de création, au niveau international (comme l'Ipbes ${ }^{1}$, qui prend modèle sur le Groupe Intergouvernemental d'Experts sur le Climat) et au niveau national (le ministère de l'Écologie a présenté en mai 2011 une nouvelle stratégie nationale pour la biodiversité ; la direction de la nature et des paysages du ministère de l'Écologie a récemment été transformée en Direction de l'Eau et de la Biodiversité). Ces institutions mobilisent des concepts nouveaux, comme ceux de « nature ordinaire », de « service écosystémique » ou de « trame verte et bleue », sur lesquels les contributions à ce numéro reviendront plus en détails.

Ce mouvement d'institutionnalisation de la biodiversité exige ainsi un regard renouvelé de la part des Sciences Humaines et Sociales. Loin d'appliquer un modèle préexistant, il témoigne en effet du développement de formes expérimentales d'action publique dans le cadre d'une alerte environnementale. Les concepts et les institutions émergentes manifestent l'extension d'une visée 
d'inventaire et d'expertise du vivant à de très larges échelles spatiales et temporelles (Höhler and Ziegler, 2010) qui questionnent les rapports entre science et politique. En effet, à l'instar du climat (Miller and Edwards, 2001 ; Demeritt, 2001), la science ne joue pas ici un rôle en amont ou à côté des processus proprement politiques mais est étroitement imbriquée dans la genèse et l'expérimentation des nouvelles institutions de la biodiversité. La biodiversité fournit alors un cas d'école pour observer et analyser les mécanismes de "l'institutionnalisation en train de se faire » d'une gouvernance internationale de l'environnement. Ce numéro vise à prendre un recul critique sur la constitution de catégories, standards et instruments formant les « allant de soi » qui sont au fondement de la prise en charge publique de la biodiversité et de son institutionnalisation. On entend ici la notion d'institutionnalisation dans une acception large (Tournay, 2009) puisqu'il s'agit précisément de comprendre les processus conduisant à la constitution et au maintien des institutions de la biodiversité au sens classique du terme. Or, qu'il s'agisse de versions pessimistes ou optimistes du futur de la nature et de notre place en son sein (Brown, 2003 et 2006 ; Tournay et Leibing, 2010), les scénarios de la biodiversité jouent un rôle structurant dans le devenir de l'action collective et dans la solidification de ses instruments et dispositifs (Lascoumes et le Galès, 2005). Il importe alors de tracer leur contenu, leurs origines et leur extension.

La notion de scénario ne réfère pas simplement à une catégorie analytique (« expectation » en anglais) développée récemment en sciences sociales. Il s'agit aussi, et avant tout, d'une notion « indigène », d'un concept largement mobilisé et travaillé par les acteurs eux-mêmes. C'est là sans nul doute une caractéristique forte du type de travail politique qui s'effectue autour de la biodiversité (comme autour du climat), par contraste avec ce qui s'est joué ou se joue toujours autour des questions sanitaires. Dans les deux cas, les acteurs sont en effet confrontés à des incertitudes majeures qui portent autant sur les mécanismes biologiques à l'œuvre que sur les pratiques humaines en jeu, témoignant de l'extension d'une société du risque (Beck, 1992) dans laquelle les experts ne peuvent plus être « formels » (Theys et Kalaora, 1992). Santé publique et problèmes environnementaux soulèvent la question cruciale de l' «agir dans l'incertain » (Callon, Lascoumes, Barthe, 2001). Mais la montée des préoccupations de santé publique a été l'occasion d'un travail collectif sur la notion de précaution qui a été largement documentée par les sciences politiques et - quoique moins largement - par la sociologie (Barbier et Granjou, 2005), alors qu'il semble que les problèmes environnementaux globaux comme le climat et la biodiversité ${ }^{2}$ alimentent, quant à eux, une élaboration collective autour des notions de prospective et de scénario qu'il importe de pointer et d'analyser.

L'idée selon laquelle il importe désormais de penser les « écologies futures » (Mermet, 2005), se retrouve dans plusieurs initiatives récentes. Imaginer l'évolution de la biodiversité jusqu'en 2030 en élaborant divers scénarios est la mission d'un groupe de travail constitué par la Direction de l'Eau et de la Biodiversité en 2010. Certes, il convient de ne pas faire preuve d'une trop grande naïveté face à ces entreprises de prospective et de scénarisation, dont nombre de participants eux-mêmes sont conscients qu'elles ne visent 
pas seulement à prédire l'avenir mais, aussi, à rassembler des individus d'horizons différents pour discuter, à la même table, des actions à engager présentement.

La question de la scénarisation prend une importance croissante dans la communauté des sciences de la biodiversité. La capacité à simuler la manière dont la biodiversité peut évoluer en fonction des différents programmes politiques envisageables dans un contexte de changements globaux apparaît en effet de plus en plus cruciale. À la suite de l'exemple du climat, la biodiversité témoigne d'un processus de normalisation du recours à la science pour l'action politique et de la stabilisation de technologies visant à construire des convictions « objectives » quant aux futurs de la planète et à l'action qu'il convient de mener. Les écologues sont ainsi incités aujourd'hui à se lancer dans l'élaboration de modèles prédictifs et de scénarios des changements de la biodiversité pour le $\mathrm{XXI}^{\mathrm{e}}$ siècle ${ }^{3}$. Comme dans le cas de la modélisation du climat (Demeritt, 2001), un glissement sémantique s'observe entre simulation et prédiction : les deux termes sont souvent utilisés comme synonymes, ce qui laisse à penser que les modélisateurs de la biodiversité sont ou seront capables de prédire l'avenir de la biodiversité.

Les « scénarios de la biodiversité » se situent au cœur du mouvement actuel de refonte et de développement de la gouvernance environnementale. Dans bien des arènes hybrides où se joue l'institutionnalisation de la biodiversité, ils semblent appelés à jouer un rôle d' " objet-frontière » (Star et Griesemer, 1989) ${ }^{4}$ entre les différentes communautés de pratiques concernées, permettant à des acteurs politiques et administratifs, des repré- sentants de firmes et d'industries, des représentants professionnels (agriculteurs par exemple), des représentants d'associations et d'ONG, des scientifiques et des acteurs médiatiques de se rencontrer et de dialoguer autour de futurs pensés, imaginés et négociés collectivement. Le dernier Cahier technique publié par la Convention sur la Diversité Biologique en 2010 ( $n^{\circ} 50$ ) s’intitule ainsi « Biodiversity scenarios : projections of $21^{\text {st }}$ century change in biodiversity and associated ecosystem services ». Ce document établit et commente une liste de scénarios pour le futur, en soulignant les degrés d'incertitude variables qui les entachent. Il témoigne d'une rhétorique propre au travail de scénarisation, où la mise en avant de l'incertitude fonde paradoxalement la puissance de l'alerte et relance simultanément la nécessité voire l'urgence du travail de scénarisation. Et si l'institutionnalisation de la biodiversité reposait justement sur la capacité de ses institutions à construire des futurs possibles qui tentent d'intégrer la prévision de l'imprévisible, dans une boucle où incertitude et scénarisation s'alimentent et se relancent mutuellement?

C'est d'abord un scénario d'alerte référant à la disparition prochaine d'espèces, d'habitats, de paysages (voire de pratiques et de modes de vie) qui est au fondement de la mise à l'agenda de la biodiversité dans les années 1980 et $1990^{5}$. Or aujourd'hui, le succès de la notion de « service écosystémique» introduit une tension structurante entre ce scénario d'alerte et l'espoir de disposer de compétences et de techniques qui garantissent l'avenir de la société humaine en bonne harmonie avec les autres « êtres de nature ». Le concept de services écosystémiques a été popularisé par la parution du Millenium Ecosystem Assessment 
(Evaluation des Ecosystèmes pour le Millénaire) en 2005, co-rédigé par plusieurs centaines de chercheurs. Les "services écosystémiques 》 désignent des fonctions remplies par les écosystèmes et utiles à l'homme. Ils relaient l'idée que les écosystèmes et la biodiversité doivent être protégés moins à cause de leur valeur intrinsèque que parce qu'ils offrent des services essentiels à la vie humaine - comme la régénération des sols ou le cycle de l'eau. Pour les défenseurs de la notion de service - taxés d'utilitarisme et d'anthropocentrisme par ses détracteurs-, la biodiversité ne peut donc plus dès lors être considérée comme un luxe fragile, puisqu'elle a directement à voir avec le bien-être et même la survie de l'humanité ${ }^{6}$. En somme, bien au-delà d'un programme «conservationniste », cette notion semble réussir à cristalliser une alliance inédite entre les enjeux environnementaux (sauvegarde des espèces, problèmes de pollution etc.), et des enjeux socio-économiques d'envergure comme la croissance de la population, l'accès à l'eau et à l'alimentation, la pauvreté, la santé publique, les inégalités NordSud etc. La notion de service porte donc avec elle la vision d'une société consciente de l'ensemble de ses interactions avec la nature dans une forme de transparence et de réflexivité environnementales (Bess, 2003), ainsi que celle d'une société gestionnaire et comptable, où il serait possible de calculer la valeur des unités de biodiversité et de compenser leurs pertes par des actions d'égale valeur. C'est à ces scénarios au fondement de la prise en charge collective de la biodiversité, et notamment à la tension entre la reformulation d'un scénario d'alerte dans l'idée de « sixième extinction » et l'émergence de technologies de maîtrise, que les contributions à ce numéro de Quaderni sont consacrées.
Isabelle Mauz analyse la relance des inventaires naturalistes, entreprises scientifiques collectives visant à recueillir, sur le terrain, des échantillons d'espèces connues et inconnues. Elle montre que pour ces inventaires naturalistes, longtemps méprisés au profit d'autres pratiques et disciplines jugées plus en pointe, l'idée de $6^{\mathrm{e}}$ extinction agit comme une "fiction essentielle » contribuant à affirmer leur nécessité et leur urgence. L'hypothèse d'une $6^{\mathrm{e}}$ extinction se trouve en retour renforcée et relayée par la relance des inventaires naturalistes.

Elizabeth Robbin nous propose une vue d'ensemble historique de l'émergence des préoccupations pour l'érosion de la biodiversité. Elle présente une série de moments de crise ayant contribué à solidifier la notion de diversité du vivant en même temps que les systèmes de savoir et d'expertise qui s'attachent à la documenter ainsi que certaines grandes institutions internationales qui entendent lutter contre la menace de son érosion. Elle interroge ainsi la place prééminente occupée aujourd'hui par les pays du Nord et par l'écologie dans la connaissance de la biodiversité.

Elsa Faugère et Olivier Pascal s'attachent à interroger la constitution d'une opinion publique autour de l'idée d'extinction et d'érosion de la biodiversité. En étudiant les acteurs et les motifs de la médiatisation de grandes expéditions naturalistes et en montrant leur évolution sur les trente dernières années, ils proposent une analyse originale de la professionnalisation du journalisme environnemental et de ses effets sur la création des « publics » de la biodiversité.

Mais si l'institutionnalisation de la biodiversité 
s'ancre dans un scénario alarmiste voire proprement apocalyptique - avec le spectre de la disparition de la vie sur Terre telle que nous la connaissons et, en particulier, de l'humanité -, elle s'accompagne simultanément d'entreprises de mise en gestion esquissant des scénarios de maîtrise. Même si ces deux types de scénarios sont la plupart du temps liés dans les instruments, les dispositifs et les institutions mis en place, la deuxième partie du numéro met l'accent sur ces scénarios et ces technologies de maîtrise.

Michel Daccache s'intéresse au développement d'une entreprise d'économisation de la biodiversité. Il propose d'interroger les arènes et les conditions sociales de la fabrication d'outils de rationalisation économique de la biodiversité. À partir d'une approche ethnographique, il montre combien la biodiversité agit aussi comme opérateur de rassemblement de mondes sociaux - scientifique, politique mais aussi de plus en plus économique - au travers d'acteurs aux profils bien particuliers.

Florian Charvolin, Raphaël Mathevet et Ruppert Vimal étudient l'émergence et la mise en pratiques d'un instrument de gouvernement inédit au niveau national, la Trame verte et bleue (TVB). Dans la lignée du Grenelle de l'environnement, ce dispositif propose une approche de gestion fondée sur les notions de continuité écologique et de nature ordinaire, qui rompent avec le principe de zonage et de sanctuarisation à l'œuvre dans la mise en place de parcs et de réserves. Les divers dispositifs d'expertise collective soutenant la concrétisation de la Trame verte et bleue contribuent à imaginer un «public putatif» dont les auteurs explorent les contours.
Enfin, Chunglin Kwa et Mieke van Hemert nous proposent une réflexion d'ensemble sur les projets de manipulation artificielle de l'environnement (geo-engineering), dont relève l'ingénierie écologique dans le cas de la biodiversité?. À partir de l'exemple d'un rapport récent de la Royal Society favorable à une relance raisonnée et encadrée de la recherche sur la manipulation du climat, ils analysent les diverses techniques envisagées afin d'en souligner les risques, notamment sur la biodiversité. À la frontière entre science et science-fiction, ils interrogent une direction originale du travail d'institutionnalisation d'une gouvernance environnementale dans son rapport particulièrement optimiste aux sciences et aux techniques. 


\section{$R \cdot E ́ E \cdot F \cdot E \cdot R \cdot E \cdot N \cdot C \cdot E \cdot S$}

M. BARBIER et C. GRANJOU, « Quand l'expertise construit la précaution. Le cas des maladies à prions » in Droit et Société, n 60, 2005, pp. 331-352.

U. BECK, Risk Society. Towards a new modernity. London, Sage Publications, U., 1992.

M. BESS, The Light-Green Society : Ecology and Technological Modernity in France, 1960-2000, The University of Chicago Press, 2003.

N. BROWN, « Hope against hype : Accountability in biopasts, presents and futures $\gg$ in Science Studies, 16/2, 2003, pp. 3-21.

N. BROWN, « Shifting tenses-from 'regimes of truth' to 'regimes of hope' », SATSU Working Paper, $\mathrm{n}^{\circ}$ 30, 2006, pp. 1-17.

M. CALLON, P. LASCOUMES et Y. Barthe, 2001. Agir dans un monde incertain. Essai sur la démocratie technique. Paris, le Seuil, 2001.

D. DEMERITT, "The Construction of Global Warming and the Politics of Science" in Annals of the Association of American Geographers vol. 9, 2001, pp. 307-337.

S. HÖHLER and R. ZIEGLER, "Nature's Accountability : Stocks and Stories" in Science as Culture, vol. 19, $\mathrm{n}^{\circ}$ 4, 2010, pp. 417-430.

P. LASCOUMES et P. LE GALÈS, Gouverner par les instruments, Paris, Presses de Science Po, 2005.

L. MERMET (dir.), Étudier des écologies futures. Un chantier ouvert pour les recherches prospectives environnementales. PIE-Peter Lang Ecopolis vol. 5, 2005.

C.A. MILLER and P. N. EDWARDS. Changing the atmosphere. Expert knowledge and environmental governance. MIT, Cambridge, Massachu- setts London, England, 2001.

S. L. STAR and J.R. GRIESEMER, J. R., "Institutional ecology « translations » and boundary objects: amateurs and professionals in Berkeley's Museum of vertebrate zoology, 1907-39" in Social Studies of Science vol. 19, n 3, 1989, pp. 387-420.

D. TAKACS, Philosophies of Paradise. The Idea of Biodiversity. Johns Hopkins University Press, Baltimore, London, 1996.

V. TOURNAY, Vie et mort des agencements sociaux. De l'origine des institutions, Paris, Presses Universitaires de France, 2009.

V. TOURNAY et A. Leibing A. (dir), Technologies de l'espoir. Les débats publics autour de l'innovation médicale - un objet anthropologique à définir. Québec : Presses Universitaires de Laval, 2010.

J. THEYS et B. KALAORA (Eds.), La Terre outragée. Les experts sont formels, Paris, éditions Autrement, 1992. 
$\mathrm{N} \cdot \mathrm{O} \cdot \mathrm{T} \cdot \mathrm{E} \cdot \mathrm{S}$

1. Intergovernmental Platform on Biodiversity and Ecological Services.

2. Par rapport à la question du climat dont la prise en charge repose aujourd'hui sur une mobilisation, des institutions (notamment le Giec) et des technologies davantage rodées, la biodiversité met au premier plan une tension entre des modes d'action globalisés et des approches nationales ou plus locales; elle pose aussi la question de la définition d'indicateurs et de métriques universelles qui puissent jouer un rôle similaire à la « tonne-équivalent carbone » ou à la température moyenne mondiale dans le domaine du changement climatique.

3. Citons deux exemples seulement. En France, la FRB a lancé en 2010 son premier appel à projets, intitulé « scénarios et modélisation pour la biodiversité ». Actuellement, modéliser la biodiversité et élaborer des scénarios apparaît comme la première des dix priorités de recherche listées par la FRB. Au niveau européen, l'EPBRS (European Platform for Biodiversity Research Strategy) a récemment adopté une stratégie de recherche dans laquelle les nouvelles approches de modélisation apparaissent parmi les ruptures conceptuelles et technologiques majeures qui ont marqué la recherche sur la biodiversité et les services écosystémiques dans la dernière décennie.

4. Un objet frontière est un objet capable de s'adapter et d'être approprié selon différents points de vue, tout en étant suffisamment robuste pour maintenir une identité stable.

5. Selon un diagnostic récent (tiré du Millenium Ecosystem Assessment : cf. infra), $60 \%$ des écosystèmes sont dégradés ou utilisés de manière non durable. Ce diagnostic, qui fait autorité dans la communauté scientifique autant que politique, est très fréquemment repris dans la littérature officielle.

6. Un responsable du MEA que nous avons interviewé commente ainsi la notion de service écosystémique :

"it was a new way of thinking about linking the environment and people [...] which has led to a recognition that the environment needs to be valued because of the benefits it provides to people rather than as a vulnerable luxury".

7. Que l'on peut définir comme la gestion, la modification et la conception par l'homme de milieux écosystémiques à partir de connaissances provenant de l'écologie. 
